\title{
SETS OF NATURAL NUMBERS WITH NO MINIMAL ASYMPTOTIC BASES
}

\author{
PAUL ERDÖS AND MELVYN B. NATHANSON
}

\begin{abstract}
The set $A$ of natural numbers is an asymptotic basis for $S$ if the sets $S$ and $2 A$ eventually coincide. An asymptotic basis $A$ for $S$ is minimal if no proper subset of $A$ is a basis for $S$. Sets $S$ are constructed which possess infinitely many asymptotic bases, but no minimal asymptotic basis.
\end{abstract}

Let $A$ and $S$ be subsets of the natural numbers $\mathbf{N}=\{0,1,2,3, \ldots\}$, and let $2 A$ denote the sum set $\left\{a+a^{\prime} \mid a, a^{\prime} \in A\right\}$. Then $A$ is an asymptotic basis (of order 2) for $S$ if the sets $2 A$ and $S$ eventually coincide, that is, if the symmetric difference $(S \backslash 2 A) \cup(2 A \backslash S)$ is finite. The asymptotic basis $A$ for $S$ is minimal if no proper subset of $A$ is an asymptotic basis for $S$. Minimal asymptotic bases for the natural numbers have been investigated by Erdös, Härtter, Nathanson, and Stöhr [1]-[6], [8], [9], [14]. The simplest example of a minimal asymptotic basis for $\mathbf{N}$ is the set consisting of all finite sums of distinct powers of 3 (Nathanson [8]). Of course, a set, such as $\left\{2^{n}\right\}_{n=0}^{\infty}$, which has no asymptotic basis certainly has no minimal asymptotic basis. Let us call two sets asymptotically equivalent if their symmetric difference is finite, and asymptotically inequivalent if their symmetric difference is infinite. The object of this note is to construct a set $S$ which has infinitely many asymptotically inequivalent asymptotic bases, but no minimal asymptotic basis.

Notation. Lower case letters denote natural numbers, and upper case letters denote sets of natural numbers. Let $A+B=\{a+b \mid a \in A, b \in B\}$. Let $[a, b]$ denote the interval of integers $n$ such that $a \leqslant n \leqslant b$.

LEMMA. Let $p_{1}=2<p_{2}<p_{3}<\ldots$ be the sequence of primes in their natural order. Let $m_{2}<m_{3}<\ldots$ be an increasing sequence of positive integers such that $p_{k} p_{k-1} \mid m_{k}$ for $k \geqslant 2$ and $m_{k+1}>2 m_{k}+p_{k} p_{k+3}$. Let $C_{k}=\{c \in$ $\left.\left[m_{k}, m_{k+1}\right] \mid c \equiv 0\left(\bmod p_{k}\right)\right\}$, and let $C=\cup_{k=2}^{\infty} C_{k}=\left\{c_{i}\right\}_{i=0}^{\infty}$, where $c_{0}<c_{1}$ $<c_{2}<\ldots$ Then $C$ is an asymptotic basis for $\mathbf{N}$ such that

(I) $\lim \sup _{i \rightarrow \infty}\left(c_{i+1}-c_{i}\right)=\infty$, and

(II) $C \backslash F$ is an asymptotic basis for $\mathbf{N}$ for every finite set $F$.

Proof. Clearly, $c_{i+1}-c_{i} \rightarrow \infty$ as $i \rightarrow \infty$.

Received by the editors February 14, 1977.

AMS (MOS) subject classifications (1970). Primary 10L05, 10L10, $10 \mathrm{J99}$.

Key words and phrases. Addition of sequences, minimal bases, sum sets. 
Let $k>5$. Let $n \in\left[m_{k}+m_{k-2}, m_{k+1}\right]$. Choose $r \in\left[1, p_{k}\right]$ so that

$$
c=m_{k-3}+r p_{k-3} \equiv n \quad\left(\bmod p_{k}\right) \text {. }
$$

Then $c \in C_{k-3}$, since $c \equiv 0\left(\bmod p_{k-3}\right)$ and $m_{k-3}<c \leqslant m_{k-3}+p_{k} p_{k-3}<$ $m_{k-2}$. Moreover, $n-c \equiv 0\left(\bmod p_{k}\right)$ and $m_{k}<\left(m_{k}+m_{k-2}\right)-\left(m_{k-3}+\right.$ $\left.p_{k} p_{k-3}\right)<n-c<m_{k+1}$, hence $n-c \in C_{k}$. Therefore, $n=(n-c)+c \in$ $C_{k}+C_{k-3}$.

Let $n \in\left[m_{k}, m_{k}+m_{k-2}\right]$. Choose $r \in\left[1, p_{k-1}\right]$ so that

$$
c=m_{k-2}+r p_{k-2} \equiv n \quad\left(\bmod p_{k-1}\right) \text {. }
$$

Then $c \in C_{k-2}$ and $n-c \in C_{k-1}$, hence $n=(n-c)+c \in C_{k-1}+C_{k-2}$. Therefore, for every $k \geqslant 5$ we have

$$
\left[m_{k}, m_{k+1}\right] \subset 2\left(C_{k} \cup C_{k-1} \cup C_{k-2} \cup C_{k-3}\right) .
$$

This implies that $C \backslash F$ is an asymptotic basis for $\mathbf{N}$ for every finite set $F$. This proves the Lemma.

THEOREM. There exist sets $S$ with infinitely many asymptotically inequivalent asymptotic bases, but no minimal asymptotic basis.

Proof. Let $C=\left\{c_{i}\right\}_{i=0}^{\infty}$ be an asymptotic basis for $\mathbf{N}$ satisfying conditions (I) and (II) of the Lemma. It follows from (II) that for every $q$ there is an integer $\phi(q)$ such that, if $n \geqslant \phi(q)$, then $n=c_{i}+c_{j}$ with $i, j \geqslant q$. Let

$$
S=\left\{2 c_{i}+1\right\}_{i=0}^{\infty} \cup\{2 n\}_{n=0}^{\infty} \text {. }
$$

We shall construct infinitely many asymptotically inequivalent bases for $S$. For $t \in \mathbf{N}$, let

$$
A_{q}(t)=\{t\} \cup\left\{2 c_{i}+1-t\right\}_{i=q}^{\infty},
$$

where $q$ is chosen large enough that $2 c_{q}+1 \geqslant t$. Clearly,

$$
2 c_{i}+1=t+\left(2 c_{i}+1-t\right) \in 2 A_{q}(t)
$$

for all $i \geqslant q$. If $n>\phi(q)$, then $n-1+t=c_{i}+c_{j}$ with $i, j \geqslant q$, hence

$$
2 n=\left(2 c_{i}+1-t\right)+\left(2 c_{j}+1-t\right) \in 2 A_{q}(t) \text {. }
$$

It follows that the set $A_{q}(t)$ is an asymptotic basis for $S$. Moreover, for $s \neq t$ the bases $A_{q}(s)$ and $A_{q}(t)$ are asymptotically inequivalent, since

$$
\begin{aligned}
{\left[2 c_{i-1}+2,2 c_{i}+1\right] \cap A_{q}(s) } & =2 c_{i}+1-s \\
& \neq 2 c_{i}+1-t=\left[2 c_{i-1}+2,2 c_{i}+1\right] \cap A_{q}(t)
\end{aligned}
$$

for all sufficiently large $i$. But $A_{q}(t) \underset{\not}{\supsetneqq} A_{q+1}(t)$, so none of the sets $A_{q}(t)$ is a minimal basis for $S$.

Now let $A$ be any asymptotic basis for $S$. Since $S$ contains infinitely many odd numbers, then $A$ must contain both even and odd numbers. But the difference between consecutive odd elements of $S$ is $\left(2 c_{i+1}+1\right)-\left(2 c_{i}+1\right)$ $=2\left(c_{i+1}-c_{i}\right)$, which tends to infinity by condition (I) of the Lemma. This implies that $A$ contains either exactly one odd element or exactly one even element. Let $t$ be the unique odd (resp. even) element of $A$. Since $2 A$ contains 
all but finitely many of the odd integers $2 c_{i}+1$,it follows that $A$ contains all but finitely many of the even (resp. odd) integers $2 c_{i}+1-t$, and at most a finite number of other even (resp. odd) integers. Thus, $A$ must be of the form

$$
A=\{t\} \cup\left\{2 c_{i}+1-t\right\}_{i=q}^{\infty} \cup F=A_{q}(t) \cup F,
$$

where $F$ is a finite set. Thus, every asymptotic basis for $S$ contains a basis of the form $A_{q}(t)$, no subset of which is a minimal asymptotic basis for $S$. Therefore, there does not exist a minimal asymptotic basis for $S$. This proves the Theorem.

REMARK. It is not known for what infinite sets there exist minimal asymptotic bases, nor even asymptotic bases. Indeed, very little is understood about the general problem of the decomposition of sets $S$ in the form $S=A+B$, where $|A| \geqslant 2$ and $|B| \geqslant 2$. This problem has been studied by Mann, Ostmann, Sárközi and Szemerédi [7], [10] [13].

\section{REFERENCES}

1. P. Erdös and E. Härtter, Konstruktion von nichtperiodischen Minimalbasen mit der Dichte $\frac{1}{2}$ für die Menge der nichtnegativen ganzen Zahlen, J. Reine Angew. Math. 221 (1966), 44-47.

2. P. Erdös and M. B. Nathanson, Maximal asymptotic nonbases, Proc. Amer. Math. Soc. 48 (1975), 57-60.

3. , Oscillations of bases for the natural numbers, Proc. Amer. Math. Soc. 53 (1975), 253-258.

4. Nonbases of density zero not contained in maximal nonbases, J. London Math. Soc. (2) 15 (1977), 403-405.

5. E. Härtter, Ein Beitrag zur Theorie der Minimalbasen, J. Reine Angew. Math. 196 (1956), 170-204.

6. __ Eine Bemerkung über periodische Minimalbasen für die Menge der nichtnegativen ganzen Zahlen, J. Reine Angew. Math 214/215 (1964), 395-398.

7. H. B. Mann, Addition theorems: The addition theorems of group theory and number theory, Interscience, New York, 1965.

8. M. B. Nathanson, Minimal bases and maximal nonbases in additive number theory, J. Number Theory 6 (1974), 324-333.

9. __ s-maximal nonbases of density zero, J. London Math. Soc. (2) 15 (1977), 29-34.

10. H.-H. Ostmann, Additive Zahlentheorie, Springer-Verlag, Berlin and New York, 1968.

11. A. Sárközy, Über total primitive Folgen, Acta Arith. 8 (1962/63), 21-31.

12. __ Uber reduzible folgen, Acta Arith. 10 (1964/65), 399-408.

13. A. Sárközy and E. Szemerédi, On the sequence of squares, Mat. Lapok 16 (1965), 76-85. (Hungarian).

14. A. Stöhr, Gelöste und ungelöste Fragen über Basen der naturlichen Zahlenreihe. I, II, J. Reine Angew. Math. 194 (1955), 40-65; 111-140.

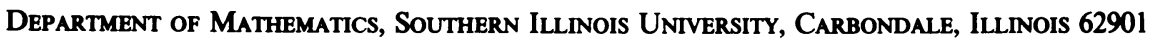

Current address (M. B. Nathanson): Department of Mathematics, Harvard University, Cambridge, Massachusetts 02138 